Editorial

\title{
Rejuvenating nerve cells in adults
}

Hui Chiu ${ }^{1,2}$ and Chieh Chang ${ }^{1}$

Like mammalian neurons, C. elegans neurons lose regeneration ability as they age, but it is not known why. $C$. elegans is a soil worm with its brain wiring diagram being mapped entirely - every connection between every nerve cell. Forty percent of genes identified in the worm genome have a counterpart in humans. Genes that allow neurons to connect with each other to form functional neuronal circuits and to regenerate themselves after injury are highly similar between worms and humans. Thus, what we learn in worms will likely be relevant to the development and regeneration of the human nervous system. The let-7 microRNA and its target, the LIN-41 tripartite motif protein, were recently shown to function as neuronal timers in worms to time the decline of the ability of neurons to regenerate as they age [1]. The progressive increase of let-7 and the progressive decrease of lin-41 in neurons provide intrinsic timing mechanism [1].
These discoveries have important implications in treating brain and spinal cord injury or neurodegenerative diseases as they show that it may be possible to improve the ability of neurons in the adult brain to regenerate after injury through therapeutic inhibition of the let-7 microRNA, and thereby restore their youthful regenerative capacity. MicroRNA does not encode a protein but rather a small RNA that imperfectly base-pairs to complementary sequences at 3' untranslated region (3'UTR) of target mRNAs in order to block gene expression [2, 3]. Approximately one third of the $C$. elegans microRNAs are evolutionarily conserved, implicating a central role for microRNAs in animals. The expression of microRNAs is either spatially restricted or temporally regulated in the nervous system. The spatially restricted expression of $l s y-6$, mir-273, and mir-71 controls left-right asymmetry in neuronal development [4-6] while the

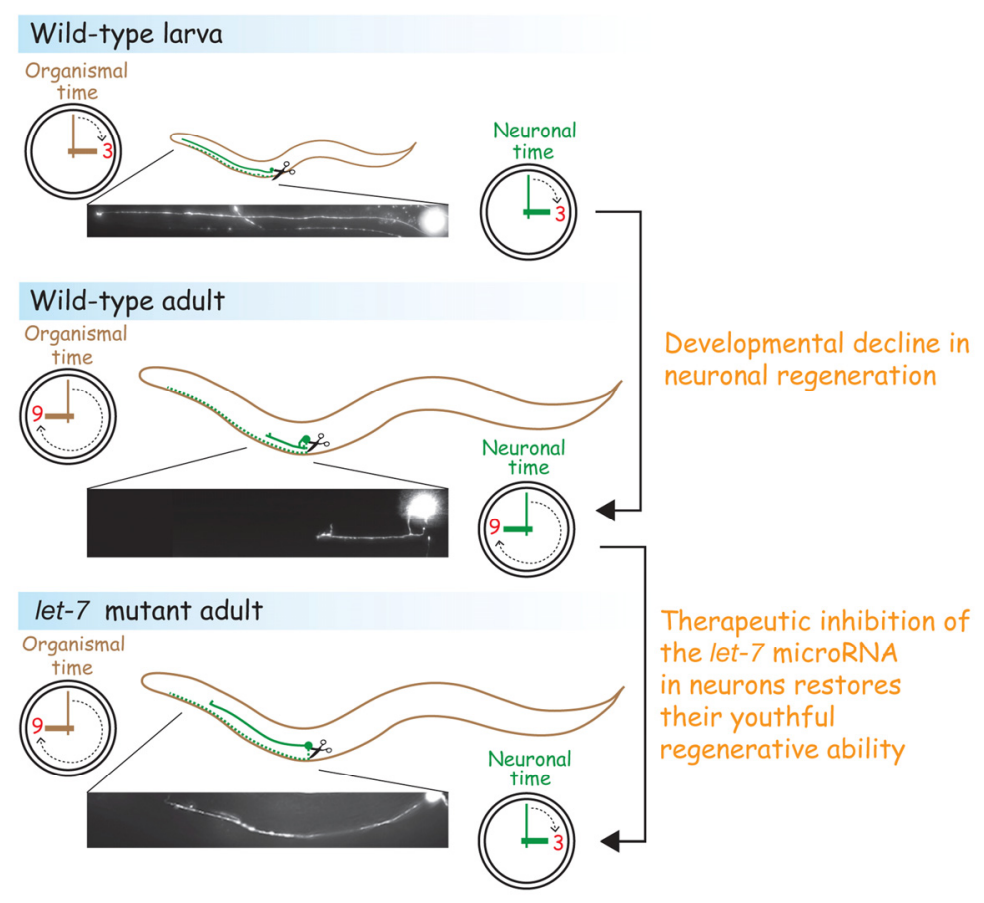

Figure 1. let-7 mutations turn back the clock on regeneration in adult neurons. Like many tissues in the body, the ability of neurons to regenerate new axons changes throughout the lifecycle, typically diminishing with age. Inhibiting let-7, or alternatively, increasing the level of its reciprocal inhibitor, lin-41, in adult neurons restored the regeneration capabilities of the larval axons. The dashed green lines indicate the disconnected axons in degeneration. The solid green lines are regenerating axons. 
temporally regulated expression of lin-4 and let-7 controls timing of neuronal connectivity and developmental decline in neuronal regeneration $[1,7]$. The let-7 microRNA represses the expression of lin-41 to inhibit anterior ventral microtubule (AVM) axon regeneration in older neurons and the effect of let-7 and lin-41 in regulating AVM axon regeneration is mediated through the LIN-29 transcription factor [1]. Since let-7 and $\operatorname{lin}-41$ genes are broadly expressed in different types of neurons [1], their roles in neuronal regeneration may be widespread. In addition to let-7, many microRNAs are also expressed in postmitotic neurons, raising the possibility that other microRNAs could also contribute to developmental decline in neuronal regeneration [1]. In C. elegans, many aged neurons display a further decline in axon regeneration. In aged AVM neurons, a reduced let-7 remains able to enhance axon regeneration so it is likely that let-7 continues to contribute to the further decline in axon regeneration in aged neurons [1]. This result also argues for a more direct role for let-7 in axon regeneration, rather than it all being a simple delay in terminal differentiation of the neuron. Like $C$. elegans neurons, mammalian neurons also suffer from the age-related decline in axon regeneration. The idea of slowing down neuronal aging to promote axon regeneration after injury is an appealing possibility. Our results suggest that one way to promote axon regeneration is to turn back the clock in old neurons via manipulation of the neuronal timing microRNA, such as let-7 [8]. Given that the let-7 microRNA sequence and its late-onset expression are highly conserved across animal phylogeny, general rules of let-7 microRNA governing neuronal regeneration in $C$. elegans are likely to be applicable in other organisms. Abundant let-7 expression in the human brain tissue appears to support this possibility.

Hui Chiu and Chieh Chang

${ }^{1}$ Division of Developmental Biology, Cincinnati Children's Hospital Research Foundation, Cincinnati, Ohio 45229;

${ }^{2}$ Present address: Division of Biology, California Institute

of Technology, Pasadena, CA 91125

Email: chieh.chang1@gmail.com

Received: 7/7/13; Published: 7/11/13

\section{REFERENCES}

1. Zou Y, Chiu H et al. Science 2013; 340:372-376.

2. Lee RC et al. Cell 1993; 75:843-854.

3. Wightman B et al. Cell 1993; 75:855-862.

4. Johnston RJ, Hobert O, Nature 2003; 426:845-849.

5. Chang $S$ et al, Nature 2004; 430:785-789.

6. Hsieh YW et al. PLoS Genet. 2012; 8:e1002864.
7. Zou Y, Chiu H et al. Sci. Signal. 2012; 5:ra43.

8. Nix P, Bastiani M, Science 2013; 340:282-283. 